\title{
USING EYE-TRACKING TO SUPPORT BIG DATA DRIVEN PROPERTY VALUATION TOOLS
}

\author{
Balamurugan Soundararaj ${ }^{1}$, Christopher Pettit $^{1}$ \\ ${ }^{1}$ City Futures Research Centre, University of New South Wales, Sydney, Australia.
}

KEY WORDS: Eye-Tracking, Automated Valuation Models, Hedonic Price Modelling.

\begin{abstract}
:
Building and using large scale, Automated Valuation Models (AVM) is one of the key multi-disciplinary pursuits in the study of cities and their economies. The methods used in building these AVMs such as 'hedonic price modelling' require a 'co-design' approach which needs significant collaboration and feedback between the modellers and the users of these models. The success of this collaborative approach depends crucially on our ability to capture the inputs and feedback from users without the bias and uncertainties present in traditional data collecting methods. In this paper, we explore and demonstrate the use of 'eye-tracking' technology in devising an objective methodology for collecting user feedback for co-design exercises. We employed a remote eye tracker in conjunction with traditional questionnaires to capture the decision making process of participants as buyers while selecting a property among a set of available options. We then compared the factors they reported to be important in their decisionmaking process to the factors they actually considered when looking at property listings. In our experiments, we found that pictures and maps captured more than $95 \%$ of the attention from buyers compared to the descriptive or statistical information showing the significance of the interface and medium of the valuation process. When responding to questionnaires, participants as property buyers reported that the attributes of a property such as number of beds, baths, quality of construction from pictures and location are equally important in selecting one over others. In contrast, when measured by an eye-tracker, we found that the participants gave significantly more attention to the quality of construction and location of the property compared to other factors. These preliminary results, though not definitive, demonstrate the value and usefulness of eye-tracking as a technique for capturing and measuring the factors that influence the desirability and in turn the price of a property. This methodology when controlled for characteristics of the participants, the properties and the medium of communication has the potential to help us to identifying and quantifying the relevance of parameters during property valuation and hence improve the accuracy and effectiveness of the corresponding hedonic price models.
\end{abstract}

\section{INTRODUCTION}

Large scale valuation of properties using big-data driven tools is one of the key research pursuits in the fields of urban planning, smart cites, urban informatics, economics, urban management and public finance. Availability of large amounts of data has made novel methodologies such as 'hedonic price modelling' (Rosen, 1974) scalable at national level into automated valuation models (AVMs) (Pettit et al., 2020). In addition to cutting edge technologies such as machine learning, building such AVMs also involve multiple levels of feedback from the users both in terms of improving the models and in improving their usability. (Barton et al., 2015) The success of this 'Codesign' process depends crucially on the quality of the feedback and removing the bias and uncertainties in them (Pettit et al., 2014) Methods such as hedonic price models try to emulate the decision making process of property valuing professionals and hence contain uncertainty/ subjectivity in the selection and weighing of various parameters used in the model. In this work we propose and explore the usage of 'Eye-tracking' system to understand the decision making process in property valuations hence reducing the uncertainties and improving the corresponding AVMs. 'Eye-tracking' systems have been extensively used in fields such as psychology, marketing, human computer interaction and few instances in real estate (Seiler et al., 2012, Sun et al., 2021) but their use in improving urban economic models and property valuation is limited. Eye-tracking provides an excellent opportunity in developing a robust, objective and quant- itative method for capturing feedback in a co-design in addition to tradition qualitative methods. In this study, we explore the use of a remote eye-tracker in the co-design process for developing a national level AVM for Australia. The system can track where a participant has been looking in flat screen in terms of fixations and saccades - quick movement between fixations with high levels of precision and accuracy. This combined and synchronised with data on stimuli on the screen can give us insights into participant's attention, thought and decision-making processes. This research aims to use this ability to understand the parameters behind the value of a property and their relative importance as perceived by various actors. The research also aims to formalise such method into a framework which can be used in the co-design approach to developing valuation models.

\section{LITERATURE}

Although eye movement studies have been performed manually as early as 1879 , it was not since 1960 s automated, precise eye-trackers and their use became popular. As vision is one of the most important sensory stimuli for humans and is deeply connected to our neural systems, movement of eyes not only provide input to our thought processes but also acts as window to our minds. The information on what people are looking at and how their eyes move gives us clues into what and how they are thinking and feeling. Such capability to track eyes accurately and precisely also enables research studies to produce replicable results even in areas of study such as cognition and 
psychology. This has made eye-tracking one of the most popular techniques in various fields such as linguistics, economics, infancy and developmental research, learning and memory, diseases and diagnosis, psychology and decision making, user experience design and usability research, sports research, communications and organizational research etc. (Carter and Luke, 2020). Being a popular and actively developed technique, there are different types of eye trackers that use different technologies, have different accuracy and precision and are static or mobile depending on their intended purpose and the context of the experiments carried out using them. The experiments themselves can be diagnostic using images, sounds etc. or interactive using web pages, virtual reality etc. depending on the intended inference (Duchowski, 2017).

Extensive research has already been carried out in developing and using the eye-trackers in various fields mentioned above, but their use within the field of urban analytics and planning have been relatively limited. There have been substantial research in understanding the perception of urban environments using eye-trackers to measure attractiveness (Vainio et al., 2019), degree of rural or urban character (Dupont et al., 2017), green spaces (Li et al., 2020), visual preferences (Noland et al., 2017), street edges (Simpson et al., 2019) etc. There have been few studies on using head mounted eye trackers in understanding people's behaviour in transport such as cyclists in traffic (Mantuano et al., 2017) and more general inquiry into understanding how spatial information acquisition happens and the associated cognitive processes (Kiefer et al., 2017) On the usability and communication side, eye-tracking has also been used to measure and compare cartographic quality of information (Burian et al., 2018) where the importance of legends and symbols. Recently, the factors that influence the tenant's decision making process during the selection of properties to rent has been studied showing that the eye-tracking can identify few factors that are not covered in a questionnaire based methods (Sun et al., 2021). Although these research help us understand the cognitive processes in decision making there is a need for expanding these insights and bringing them back to applications in urban analytics and management.

\section{METHODOLOGY}

The primary aim of this research is to explore and demonstrate the value of eye-tracking to understand decision making behaviour of property buyers. The secondary objectives of the research threefold,

1. To check if there were any difference between the interview and eye-tracking results.

2. Explore the usefulness of eye-tracking in supporting a hedonic price modelling process

3. To gain feedback on scaling the experiment and process further.

The experiment was designed as three stages, first stage where the participants are explained the tasks they need to complete, completion of a pre-task interview and finally the experiment task. Each participant was given an assumed persona for themselves - a 'Home Buyer' from Sydney who is looking to buy a residential property to live within a budget of AUD 2.5 Million in the eastern suburb of Bondi. The participants were shown web listings for three distinct properties from the Bondi area from domain.com. Participants were then asked to select a property out of these three options from the information present in the listings. Before the online task and after the briefing, the participants were interviewed on the most important factors they consider when choosing a property along with their experience with the market and domain.com website. The participants then looked at the listings for the options one by one while their eye being tracked. Each participant was given two trials to complete the tasks where each trial took approximately 10-15 minutes. The experiment was concluded with a brief interview on the selected option and how they arrived at the selection. A total of 5 participants and 12 trials were conducted over a period of week and three of the trials were discarded for having less than $80 \%$ of gaze samples - due to interference such as eye glasses.

The eye-tracker employed was a 'Tobii Pro Spectrum' connected to a 21 inch LCD monitor with a resolution of 1920x1080 as shown in 1 . The eye-tracker had a frequency of $1200 \mathrm{~Hz}$ with a latency of $2.5 \mathrm{~ms}$ and along with a chin rest, it could track the location of participants gaze on the screen with high degree of accuracy and precision. The system is capable of dynamic stimuli such as websites and keeps track of the scroll position on the page as the participants gaze move across the webpage.

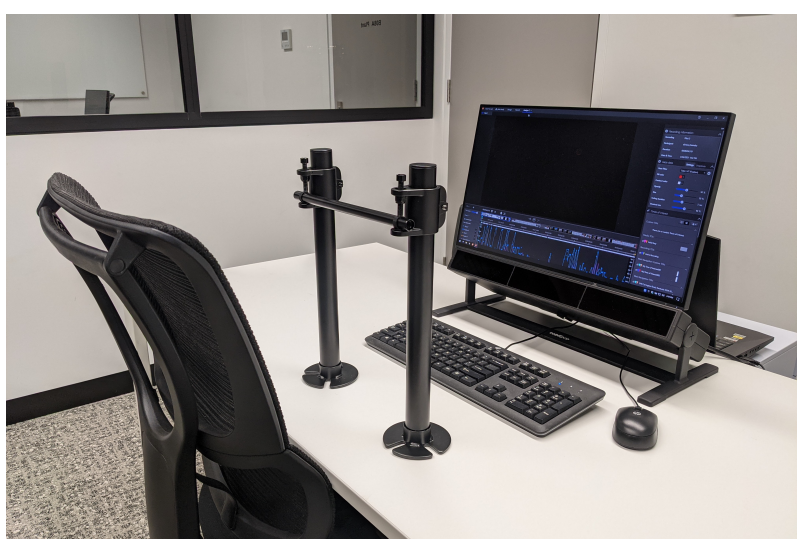

Figure 1. Hardware setup of the Eye-tracking system.

\section{RESULTS}

Although numerous analyses with varying levels of granularity can be carried out on the data collected from the trials such as gaze sequences, micro saccades etc, we primarily looked at two lines of analysis for this research.

- Visual analytics using heat maps

- Area of Interest (AOI) analysis

The cumulative map of fixations - areas where the participants looked at in a property listing is shown in Figure 2. We observed that the participants on average spent more than $95 \%$ of their time on the visual elements of the property listing such as pictures (including floor plans) and location map. Compared to the visual elements, other elements such as the number of bedrooms, bathrooms, and parking spaces and the text description of the properties are not at viewed by the participants when making their decisions. This shows the significance of the medium and format in which the information is communicated to the participants. Although we have to note that the listing website gave much more prominent position and emphasis to 


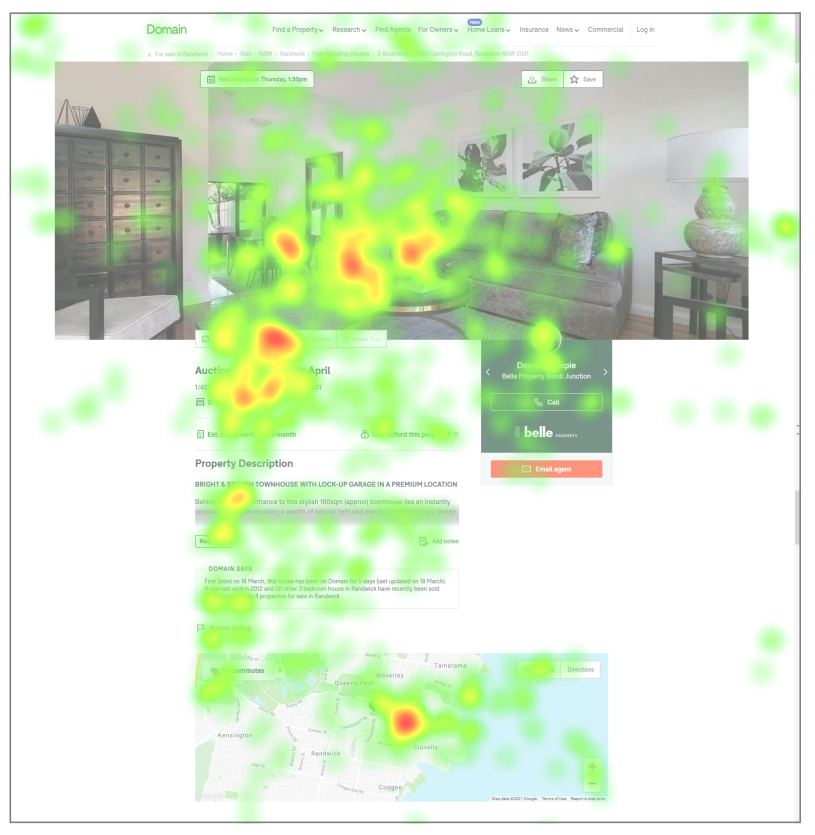

Figure 2. Heat-map showing the cumulative fixations on a property listing across all the trails.

the pictures and maps which may have influenced the participants, we also see variations in the gaze behaviour between participants. Figure 3 shows the difference between four different participants when evaluating the same property listing. We can observe that some participants have completely different gaze patterns than the rest of them where. For example, participant $\mathrm{P} 2$ is more focussed on the data and description of the property more than the pictures and maps. We can also observe difference in behaviour such as P1 reading the entire listing, $\mathrm{P} 4$ focussing mostly on the location map of the property thus showing the importance of controlling our trials for the characteristics of the participants such as experience, income and age to remove the biases created by them.
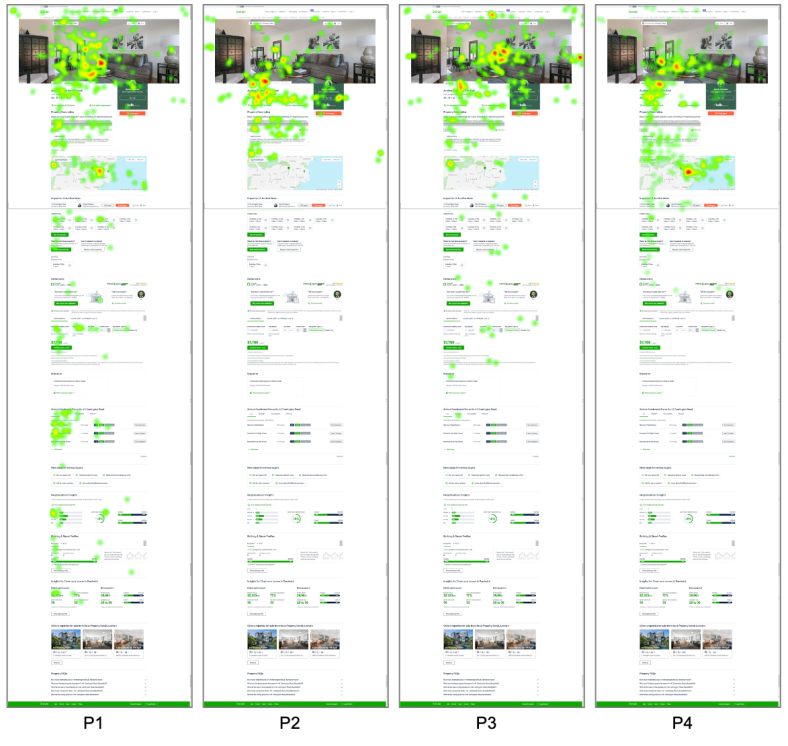

Figure 3. Comparing heat-maps of fixation between 4 different participants on the same property listing.

To compare the attention paid to the elements by the parti- cipants to the questionnaire results we first defined Area of Interests (AOI) on the listings and measured the amount of time the participant spent looking within the AOI. The results of this AOI analysis for the participants is shown along side the results from the questionnaires is shown in Figure 4. We observe that the Quality of construction and floor plan from the pictures are the most considered AOI with $75 \%$ of the time spent. Following that participants spend another $20 \%$ of the time on the interactive map evaluating the location of the property. Notably, the AOI showing the number of bedrooms in the page was not seen by any participant and the price was noticed the least in the page. This could be partly explained by the constrained set up of the experiments where the participants knew the budget beforehand and might had a fair idea on size of the property available for the price. Figure 4 shows the contrast between the results from the pre-task interview and the eye tracking on the significance of various factors that influences the participants in choosing a property. Cumulatively, participants reported to consider the factors - number of bedrooms, bathrooms, car ports, location, construction quality from photos and the price of the property almost equally but data from eye-tracking suggests otherwise. Alternatively the participants might also be gathering information on beds, baths and car ports from the pictures rather than looking at their corresponding AOIs showing a need for breaking down the pictures into further AOIs to pick up subtle indicators such as amount of light, greenery, quality, etc.

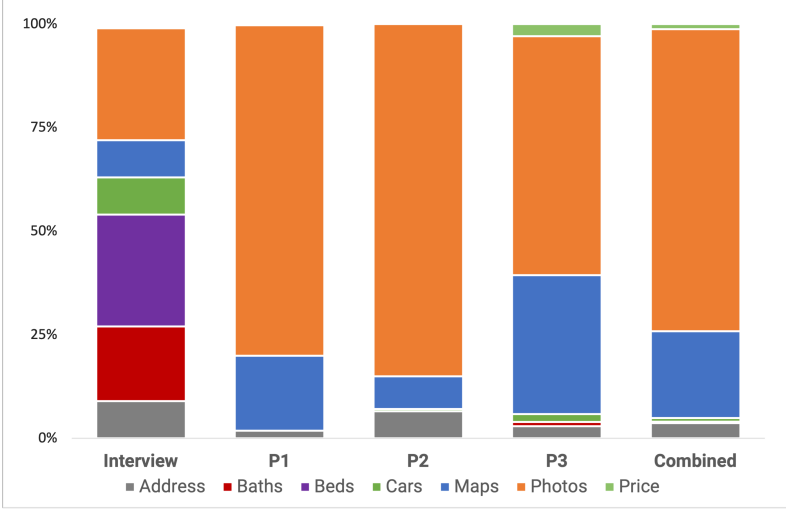

Figure 4. The percentage of time spent by participants on different Area of Interests in the listing compared to their corresponding proportion of interview answers.

\section{DISCUSSION AND CONCLUSIONS}

The study has clearly demonstrated the subjectivity and uncertainty in the factors reported by the home buyers as important for choosing a property compared to the actual attention paid by them when measure using an eye-tracker. With all things equal, participants thought they valued the factors such as number of beds, baths, parking, price etc. equally but in practice they focussed far more on the build quality and location of the property. This shows the significance of the bias when the self reported factors are used as input for automated valuation methods such as hedonic price modelling.

Although the pilot study demonstrated the value of eye-tracking data in capturing the thought process of the participants in choosing a property as home buyers, it also shows the various ways process could be improved for reliable results. First and foremost, the participant pool needs to be expanded in terms of 
size and diversity of background. It would be beneficial to include industry participants such as property valuers and lenders so that we can understand their thought process in evaluating properties. The scenarios in the pilot study with fixed price and location might have influenced the outcome of AOIs analysis. This could be mitigated by having the comparison of properties arise from a search guided by the participants themselves. We also need to take into account significant biases introduced by the optimisations carried out by the listing websites for their revenue generation. This could be removed by creating a standardised listing design or property fact sheet for the main study.

Moving forward we hope to apply the learnings from the pilot study to conduct a broader main experiment which is expected to produce outputs showing the most important factors influencing the valuation of a property and their relative perceived importance. This could be in-turn used to fine tune the large scale big-data driven hedonic price modelling process to closely reflect the ground valuations carried out by property valuers.

\section{ACKNOWLEDGEMENTS}

This work has been supported by FrontierSI, a not-for-profit company that exists to deliver major benefits to governments, industry and the community in Australia and New Zealand through the application of spatial information. This research was funded through the Cooperative Research Centre Project - Value Australia.

\section{REFERENCES}

Barton, J. E., Goldie, X. H., Pettit, C. J., 2015. Introducing a usability framework to support urban information discovery and analytics. Journal of Spatial Science, 60(2), 311-327.

Burian, J., Popelka, S., Beitlova, M., 2018. Evaluation of the cartographical quality of urban plans by eye-tracking. ISPRS International Journal of Geo-Information, 7(5), 192.

Carter, B. T., Luke, S. G., 2020. Best practices in eye tracking research. International Journal of Psychophysiology, 155, 4962.

Duchowski, A. T., 2017. Eye tracking methodology: Theory and practice. Springer.

Dupont, L., Ooms, K., Duchowski, A. T., Antrop, M., Van Eetvelde, V., 2017. Investigating the visual exploration of the rural-urban gradient using eye-tracking. Spatial Cognition \& Computation, 17(1-2), 65-88.

Kiefer, P., Giannopoulos, I., Raubal, M., Duchowski, A., 2017. Eye tracking for spatial research: Cognition, computation, challenges. Spatial Cognition \& Computation, 17(1-2), 1-19.

Li, J., Zhang, Z., Jing, F., Gao, J., Ma, J., Shao, G., Noel, S., 2020. An evaluation of urban green space in Shanghai, China, using eye tracking. Urban Forestry \& Urban Greening, 56, 126903.

Mantuano, A., Bernardi, S., Rupi, F., 2017. Cyclist gaze behavior in urban space: An eye-tracking experiment on the bicycle network of Bologna. Case studies on transport policy, 5(2), 408-416.
Noland, R. B., Weiner, M. D., Gao, D., Cook, M. P., Nelessen, A., 2017. Eye-tracking technology, visual preference surveys, and urban design: preliminary evidence of an effective methodology. Journal of Urbanism: International Research on Placemaking and Urban Sustainability, 10(1), 98-110.

Pettit, C. J., Glackin, S., Trubka, R., Ngo, T., Lade, O., Newton, P., Newman, P., 2014. A Co-design Prototyping Approach for Buiding a Precinct Planning Tool. ISPRS Journal of Photogrammetry and Remote Sensing, 2, 47-53.

Pettit, C., Shi, Y., Han, H., Rittenbruch, M., Foth, M., Lieske, S., van den Nouwelant, R., Mitchell, P., Leao, S., Christensen, B. et al., 2020. A new toolkit for land value analysis and scenario planning. Environment and Planning B: Urban Analytics and City Science, 47(8), 1490-1507.

Rosen, S., 1974. Hedonic prices and implicit markets: product differentiation in pure competition. Journal of political economy, 82(1), 34-55.

Seiler, M., Madhavan, P., Liechty, M., 2012. Toward an understanding of real estate homebuyer internet search behavior: an application of ocular tracking technology. Journal of Real Estate Research, 34(2), 211-242.

Simpson, J., Freeth, M., Simpson, K. J., Thwaites, K., 2019. Visual engagement with urban street edges: insights using mobile eye-tracking. Journal of Urbanism: International Research on Placemaking and Urban Sustainability, 12(3), 259-278.

Sun, J., Wang, Z., Dang, X., Zhang, Y., 2021. Eye-Tracking Technology in Online Real Estate Rental. Scientific Programming, 2021.

Vainio, T., Karppi, I., Jokinen, A., Leino, H., 2019. Towards novel urban planning methods-using eye-tracking systems to understand human attention in urban environments. Extended Abstracts of the 2019 CHI Conference on Human Factors in Computing Systems, 1-8. 